\title{
Anticipatory Postural Adjustments and kinematic arm features when postural stability is manipulated
}

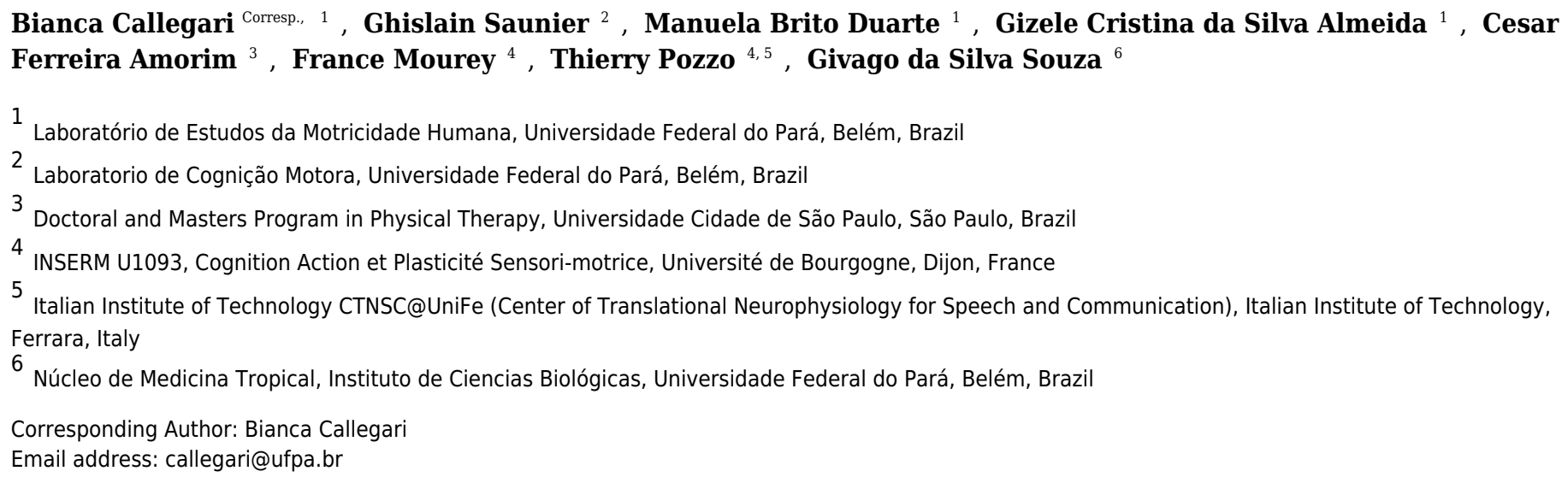

Beyond the classical paradigm that presents the Anticipatory Postural Adjustments (APAs) as a manner to create forces that counteract disturbances arising from the moving segment, during a pointing task, there is a controversial discussion about the role APAs to facilitate the movement and perform a task accurately. In addition, arm kinematics features are classically used to infer the content of motor planning for the execution and the control of arm movements. The present study aimed to disentangle the conflicting role of APAs during an arm-pointing task in which the subjects reach a central diode that suddenly turns on, while their postural stability was manipulated. Three postures were applied: Standing (Up), Sit without feet support (SitUnsup) and Sit with feet support (SitSup). We found that challenging postural stability induced an increase of the reaction time and movement duration (observed for the SitUnsup compared to SitSUp and Up) as well as modified the upper-limb velocity profile. Indeed, a greater max velocity and a shorter deceleration time were observed under the highest stability (SitSup). Thus, these Kinematics features reflect less challenging task and simple motor plan when the body is stabilized. Concerning the APAs, we observed the presence of them independently of the postural stability. Such a result strongly suggests that APAs act to facilitate the limb movement and to counteract perturbation forces. In conclusion, the degree of stability seems particularly tuned to the motor planning of the upper-limb during a pointing task whereas the postural chain (sitting vs. standing) was also determinant for APAs. 

postural stability is manipulated

3 Bianca Callegari ${ }^{1}$, Ghislain Saunier ${ }^{2}$, Manuela Brito Duarte ${ }^{1}$, Gizele Cristina da Silva Almeida ${ }^{1}$,César

4 Ferreira Amorim $^{3}$, France Mourey ${ }^{4}$, Thierry Pozzo ${ }^{4,6}$,Givago da Silva Souza 5 .

5 Laboratório de Estudos da Motricidade Humana, Federal University of Pará, Av. GeneralíssimoDeodoro 6 01, 66073-000, Belém, Pará, Brazil

7 22Laboratorio de Cognição Motora, Departamento de Anatomia, Universidade Federal do Pará, Rua 8 Augusto Correa 01, 66075-110, Belém, Pará, Brazil

9 ' ${ }^{3}$ Doctoral and Masters Program in Physical Therapy, UNICID, 448/475 Cesário Galeno St., São Paulo, 10 SP, Brazil

$11{ }^{4}$ INSERM U1093, Cognition Action et Plasticité Sensori-motrice, Université de Bourgogne Franche-

12 Comté, Dijon, France

$13{ }^{5}$ Institute of Biological Sciences, Federal University of Pará, Rua Augusto Correa 01, 66075-110, Belém, 14 Pará, Brazil

$15{ }^{6}$ Italian Institute of Technology CTNSC@UniFe (Center of Translational Neurophysiology for Speech

16 and Communication), Ferrara, Italy

\section{Corresponding author:}

18 Bianca Callegari

19 Avenida Generalissimo Deodoro, nº1, Instituto de Ciências da Saúde, 66055-240, Belém-PA,

20 Brazil

21 Phone: + 5591982210054

22 E-mail: callegaribi@uol.com.br 


\section{Abstract}

Beyond the classical paradigm that presents the Anticipatory Postural Adjustments (APAs) as a manner to create forces that counteract disturbances arising from the moving segment, during a pointing task, there is a controversial discussion about the role APAs to facilitate the movement and perform a task accurately. In addition, arm kinematics features are classically used to infer the content of motor planning for the execution and the control of arm movements.

The present study aimed to disentangle the conflicting role of APAs during an arm-pointing task in which the subjects reach a central diode that suddenly turns on, while their postural stability was manipulated. Three postures were applied: Standing (Up), Sit without feet support (SitUnsup) and Sit with feet support (SitSup). We found that challenging postural stability induced an increase of the reaction time and movement duration (observed for the SitUnsup compared to SitSUp and Up) as well as modified the upper-limb velocity profile. Indeed, a greater max velocity and a shorter deceleration time were observed under the highest stability (SitSup). Thus, these Kinematics features reflect less challenging task and simple motor plan when the body is stabilized. Concerning the APAs, we observed the presence of them independently of the postural stability. Such a result strongly suggests that APAs act to facilitate the limb movement and to counteract perturbation forces. In conclusion, the degree of stability seems particularly tuned to the motor planning of the upper-limb during a pointing task whereas the postural chain (sitting vs. standing) was also determinant for APAs. 


\section{Introduction}

Postural perturbations induced by fast arm movements in a standing condition were widely studied and generally displace the body's center of mass (COM) causing a disruption of posture. Classically, it is accepted that anticipatory postural adjustments (APAs) begin before the limb upward movement taking place to counteract the expected mechanical effects of the focal perturbation in a feedforward way (Moore et al., 1992; Santos, Kanekar \& Aruin, 2010). Authors suggest that they are generated at a high level of the central nervous system (CNS) promoting earlier changes in the activity of the postural muscles in order to compensate for a potential shift in the COM (Sijper \& Latash, 2000; Yoshida et al., 2008).

Beyond this classical paradigm that presents APAs as a manner to create forces that counteract disturbances arising from the moving segment, there is a controversial discussion about the role of present APAs. For instance, Stapley et al (1999) found that, rather than acting to stabilize the COM, APAs created necessary conditions for forward COM displacement within the base of support in the upright position(Stapley et al., 1999). Tijtag et al (2013) investigated APA in one-handed ball catching and proposed that APAs besides a stabilization of some body part are functionally link to the task specificity and ensure an active role in task goal achievement (Tijtgat et al., 2013).

Their results challenge the classical role assigned to APAs in the literature. In this paper we propose to contribute to this controversial issue by studying APA in a stable posture, where equilibrium control is minimized and thus the APA may be more related to the task performance.

While sitting, the base of support is substantially larger and the center of mass is positioned closer to the base of support than in the standing position. Thus, the task of maintaining the center of mass projection within boundaries of the base of support is less challenging and one may expect different patterns of APAs before arm pointing.

The majority of studies on the APAs have been carried out in standing adults performing various types of arm movements (Cholewicki, Polzhofer \& Radebold, 2000; Sijper \& Latash, 2000; Cecchi, Došlă \& Marini, 2001; Juras \& Słomka, 2013) and the few existing reports while sitting are somewhat incomplete and conflicting(Van Der Fits et al., 1998; Aruin \& Shiratori, 2003a; van der Heide et al., 2003; Le Bozec \& Bouisset, 2004; Cuisinier, Olivier \& Nougier, 2007). In particular, APAs are sometime absent in individuals performing reaching tasks while sitting (Moore \& Brunt, 1991; Van Der Fits et al., 1998), while other studies, where seated subjects exerted maximal force on a bar, showed APAs in trunk and hip muscles (Teyssedre et al., 
75 2000; Le Bozec \& Bouisset, 2004). Although these studies have achieved interesting results,

76 some of them did not involve lower limb muscle investigation, which are mainly responsible for

77 ankle joint mobility (i.e., tibialis anterior and soleus). Indeed, a recent review of the literature

78 showed that the effect a manipulated initial position, as the amount of feet and/or back support, or

79 leaning toward a side, for example, was not studied (Chikh et al., 2016).

80 Aruin and Shiratori (2003) found that APAs in sitting with feet support compared to

81 standing were attenuated in the leg muscles (tibialis anterior, soleus, rectus femoris, and biceps

82 femoris) but not in trunk muscles (erector spinae and rectus abdominis)(Aruin \& Shiratori,

83 2003a). However, they only compared the amount of muscle activation and not the latency within

84 a temporal window of $100 \mathrm{~ms}$ before the movement. Thus, they precluded conclusions on the

85 activation order within the direction specific response. In addition, they did not worry about

86 important aspects of the sitting posture, such as the amount of feet and/or back support. Indeed,

87 the quantity of reaction force exerted by the feet determines the center of pressure position along

88 the antero-posterior axis and the torque for the task.

89 Accordingly, the present study aimed to disentangle the conflicting role of APAs during an

90 upper-limb motion, using different postural stabilities (standing and sitting). For this, we used an

91 arm-pointing task in which the subjects reach a central diode that suddenly turns on, while their

92 postural stability was manipulated. In order to characterize the movement performance, we

93 measured the kinematic parameter and registered the latency of lower limb muscles. We

94 hypothesized that different arm kinematics features reflecting postural stability (i.e. arm shorter

95 reaction time(Papaxanthis, Pozzo \& Schieppati, 2003; Berret et al., 2009), arm velocity

96 profile(Stapley, Pozzo \& Grishin, 1998; Stapley et al., 1999) will be present during unstable

97 postures, suggesting different motor plans for the execution and the control of arm movements

98 performed in different equilibrium context. We further hypothesized that whether the main

99 concern of APA is the compensation of postural perturbations (i.e., consequences of COM

100 displacement), then the increase of body stability should induce attenuation or a modification of

101 APA patterns. On the other hand, if APA serves the preparation for forthcoming upper limb

102 movement (i.e. in order to accelerate the center of mass) to perform the task accurately, one could

103 expect the presence of similar APAs patterns independently of the equilibrium constraint.

$104 \quad$ Experimental Procedures

105 Subjects 
106

107

108

109

110

111

112

113

114

115

116

117

118

119

120

121

122

123

124

125

126

127

128

129

130

131

132

133

134

135

Ten healthy men between the ages of 21 and 28 years (mean height $1.71 \pm 0.04 \mathrm{~m}$, weight $71.8 \pm 6.2 \mathrm{~kg}$ ), with normal or corrected to normal vision, no known neurological or muscle disorders, took part in the study. All subjects were right-handed. They gave their informed consent. The study was approved by the Federal University of Pará's ethics committee (Ethical Application 46943215.0.0000.0018), before the beginning of the study.

\section{Experimental set up and Protocol}

Subjects either stood on the floor barefoot (Up) or were seated on the edge of a heightadjustable chair with (Sit Sup) or without feet on a force platform (SitUnsup). In all seated posture we preconized that the surface in contact with the chair was $30 \%$ of the thigh length (i.e., from the head to the femur to the intra-articular line of the knee). To ensure any contact of the feet with the ground we adjusted the height of the chair, in SitUnsup posture. In SitSup posture, we standardized the ground reaction force $(\mathrm{Fz})$ not to exceed $5 \%$ of the total $\mathrm{Fz}$ during stance (Fig. 1).

\section{Insert Figure 1}

Figure1. Experimental pointing task. View of the experimental set-up for the task showing a participant in final posture and the three possible targets. The central diode of the bar between the two laterals was situated exactly in front of the participants' right shoulder. Participants were asked to point their index finger at the central diode which suddenly turned on. Postures: A(Up); B (SiUnsup); C (SitSup)

They were required to keep their eyes fixed on a horizontal bar, placed in front of them at $2 \mathrm{~m}$ from the floor and $2.5 \mathrm{~m}$ from the participants' feet, with a central diode aligned with their right shoulders. Participants were asked to point with their index finger towards the central diode, which was suddenly turned on. Subjects stayed with the left arm down along the body and the right index finger pointing towards the ground, with an angle of between $30^{\circ}$ and $35^{\circ}$ between the arm and trunk. In all trials, participants were told to raise their arm as fast as possible and to start as quickly as possible after the appearance of the visual stimuli, remaining for a few seconds with their arm in the air, and to move their index finger back towards the initial starting position. Randomized blocks of ten trials in each posture were performed with a 5 minutes' rest between them.

\section{Kinematic and electromyographic recording}

A three-dimensional motion analysis system (Simi Motion), with two cameras at a 
136 sampling frequency of $120 \mathrm{~Hz}$, was used in order to record the participants' movements. The

137 participant had eight infrared reflective markers placed at the main joints of right upper limb (i.e.,

138 index; wrist; elbow and shoulder).

139 Surface electromyographic (EMG) data, from the dominant-side leg and trunk muscles 140 using disposable self-adhesive electrodes (Red Dot, $3 \mathrm{M}$ ): tibialis anterior (TA), soleus (SOL), 141 rectus femoris (RF), semitendinosus (ST) and Deltoidis anterior, were recorded using two EMG 142 devices (Emgsys 30306®, EMG System do Brazil, Brazil), with a sampling rate of $2 \mathrm{KHz}$ and a 143 frequency spectrum of 20 to $500 \mathrm{~Hz}$. The EMG signals were amplified (4,000), and digitized with 144 a 16-bit resolution. The participants' skin was prepared for $\mathrm{Ag} / \mathrm{AgCl}$ electrodes (Medtrace ${ }^{\circledR} 200$ 145 - Kendall, Canada) using Nuprep ${ }^{\circledR}$ (Weaver and Company - Aurora, United States) and alcohol. 146 The active electrodes were placed on the muscles at $20-\mathrm{mm}$ intervals, and the reference electrode 147 was placed on the spinous process of the seventh cervical vertebra based on the orientations 148 proposed by the Surface Electromyography for Non Invasive Assessment of Muscles 149 guidelines(Hermens et al., 2000).

\section{Data analysis parameters and Statistics}

\section{Kinematic data}

152 The kinematic parameters of each marker (trajectory and tangential velocity profile) were

153 analyzed using Matlab. The data on $\mathrm{x}, \mathrm{y}$ and $\mathrm{z}$ axis were filtered with a 10-Hz low-pass, second 154 order Butterworth filter. We defined the total movement duration (MD) as the time interval of the 155 index tangential velocity profile that exceeded five percent of its maximum value. Moreover, we 156 calculated the movement velocity (MV) and the reaction time (RT). Finally, we also examined 157 the differences of index tangential velocity profile in function of participant postures. For this, we 158 calculated the ratio of acceleration time to total movement duration (i.e., time to peak velocity).

\section{EMG data}

160 The data were synchronized and analyzed off-line with MatLab programs. All signals were 161 filtered with a 100-Hz low-pass, second-order Butterworth filter, and EMG signals were rectified. 162 Individual trials were viewed off-line on a monitor screen.

163 After additional visual inspection of the data, Tzero moment, as defined by the marker on 164 the finger, was considered as the instant when the tangential velocity of the marker reached $5 \%$ of 165 PV during that particular trial (Bertucco \& Cesari, 2010). After alignment, trials within each 
166 series were averaged for each subject. To quantify the anticipatory changes in the muscle activity 167 prior to movement, EMG signals were integrated from -150 with respect to Tzero (JEMG150).

168 This was further corrected for background activity, defined as the integral from -500 to $450 \mathrm{~ms}$ 169 with respect to Tzero (JEMG50) as follows:

170

$$
\int \mathrm{EMG}=\int \mathrm{EMG} 150-3 * \int \mathrm{EMG} 50
$$

The muscle latency was detected in a time window from $-450 \mathrm{~ms}$ to $+200 \mathrm{~ms}$ in relation to Tzero by a combination of computer algorithm and visual inspection of the averaged trials. The latency for a specific muscle was defined as the instant lasting for at least $50 \mathrm{~ms}$ when its EMG amplitude was greater (activation) or smaller (deactivation) than the mean of its baseline value, measured from-500 to $-450 \mathrm{~ms}$, plus 2 SD (Aruin \& Shiratori, 2003b).

\section{Statistics}

Statistical procedures were performed in RSudio (R version 3.3.2 and RStudio 1.0.136) and included repeated-measures ANOVA with Body posture (sitting whit support, sitting without support and standing) as factor. Post-hoc analyses were done with Tukey HSD tests when necessary. For all these statistical treatments, the significance level was set at $\mathrm{p}<0.05$.

\section{Results}

\section{Kinematic characteristics}

Kinematic' characteristics are summarized in Table 1. Finger displacement showed that a similar trajectory length was performed in the three posture $[\mathrm{F}(2,28.9), \mathrm{p}=0.77)]$. RT presented a main posture effect between the three postures $[\mathrm{F}(2,6.41), \mathrm{p}=0.005])$. Post-hoc test revealed that RT was higher in SitUnsup posture compared to standing and Sit Sup posture $(\mathrm{p}<0.001)$. MD also showed a main posture effect $[F(2,3.56)=0.0042]$ and the post-hoc test showed the highest MD in the SitUnsup posture, compared to the others $(p<0.001)$. No statistical differences were observed for MV of the index movement between the three postures $[\mathrm{F}(2,2.46)=0.104])$. The results also revealed a postural effect onto index tangential velocity profile $[\mathrm{F}(2,10.86)=$ 0.0003]. In Sitsup posture subjects had higher ratio of acceleration time to total movement duration comparing to Up and SitUnSup postures. 
194 the postural conditions whereas their spatial features remained similar.

195 Table 1. Comparison between Kinematic parameters.

196 Mean values kinematic parameters. Values are given as mean (SD). ${ }^{*} \mathrm{p}<0.005$ difference between

197 Sit Unsup and the two other postures. ${ }^{*} \mathrm{p}<0.005$ difference between Sit Sup and the two other 198 postures.

\section{Muscle Activation Timing between postures}

Figure 2 shows the rectified EMG data obtained for one trial for a typical subject comparing the tree postures.

\section{Insert Figure 2}

Figure 2:Raw muscle activity of a typical subject recorded during one single trial. Plotted signals were just rectified and normalized with respect to their maximum values recorded over all trials. The vertical dashed line mark finger movement onset (tzero). The time interval between $300 \mathrm{~ms}$ before movement onset was considered.Up: Upright posture; SitSup: Sit posture with contact feet support; SitUnsup: Sit unsupported posture; Muscles: DEL: Deltoideous; ST: Semitendinosus; RF: Rectus Femoris; SOLSoleous; TA: Tibialis Anterior.

Figure 3 shows the scatter of the overall dataset of the timing of muscle activations (all

211 the trials of all participants in the three protocols).

In the Upright posture, muscles participated in the APAs in the following order: the

213 proximal lower limb muscles (ST: 110ms \pm 10 and RF $80 \mathrm{~ms} \pm 20$ ) followed by the distal lower limb

214 muscles (SOL: $80 \mathrm{~ms} \pm 10$ and TA: $70 \mathrm{~ms} \pm 30$ ). While seated this order were reverse: TA and SOL

215 had earlier onset [SitSup (TA: 90ms \pm 20 and SOL: 80ms \pm 20 ) and SitUnsup (TA: 90ms \pm 10 and

216 SOL: 100ms \pm 10], followed by ST and RF [SitSup (ST: 90ms \pm 20 and RF: 80ms \pm 10 ) and

217 SitUnsup (ST: 90ms \pm 10 and RF: $80 \mathrm{~ms} \pm 10)]$.

218 Comparing the timing of muscle activation between the postures, the ANOVA showed a 219 main posture effect for $\mathrm{TA}[\mathrm{F}(2,591)=18.18, \mathrm{p}<0,000001]$ and $\mathrm{ST}[\mathrm{F}(2,591)=18.18$, 
220

221 222

$\mathrm{p}<0,000001]$. The post-hoc analysis revealed earlier activations of the ST in Upright posture ( $\mathrm{p}<$ $0.001)$ compared to both seated posture and earlier onset of TA in seated posture $(p<0.001)$ compared to Up.

\section{Insert Figure 3}

Figure 3 : Latency (activation/deactivation before tzero) for lower limb muscles. Up: Upright posture; SitSup: Sit posture with contact feet support; SitUnsup: Sit unsupported posture; Muscles: DEL: Deltoideous; ST: Semitendinosus; RF: Rectus Femoris; SOLSoleous; TA: Tibialis Anterior. ${ }^{*} \mathrm{p}<0,05$ two way anova and post hoc test (differences between SIT SUP x UP and SIT UNSUP x UP)

\section{Muscle Activation Rates and Magnitude between Postures}

To demonstrate the consistence of the results, we calculated the activation rate for each muscle in each posture, which corresponded to the percentage of trials showing significant muscle activation (burst). This corresponded to a minimal of $60 \%$ of trials with burst.

The normalized integrated electromyographic activity (EMGi) are summarized in Table 2. SOL and ST showed a similar behavior and presented a main posture effect between the three postures $[\mathrm{F}(2,6.31), \mathrm{p}=0.003])$. Post-hoc test revealed that both SOL and ST had higher APA integral in Up posture

compared to SitUnsup and SitSup $(\mathrm{p}<0.001)$. RF also showed a main posture effect $[\mathrm{F}(2,4.56)$ $=0.002]$ and the post-hoc test showed the lowest APA integral in the SitUnsup posture, compared to the others $(\mathrm{p}<0.001)$. The results also revealed a postural effect onto TA APA integral $[F(2,9.84)=0.0003]$. In Sitsup posture subjects had higher values comparing to both Up and SitUnsup postures

Insert Table 2

\section{Temporal organization of finger and knee displacements}

After processing the EMG data and observing the movement execution we decided to repeat the experiment with four additional subjects. Indeed, the visual observation of the pointing task performed with a SitUnsup posture showed a clear posterior displacement of the ankle joint (i.e knee flexion). To quantify such displacement and its relation with the upper-limb motion we 
247 recorded the kinematic of three additional markers placed on the right lower limb (i.e., $2485^{\text {th }}$ metatarsal; ankle; knee and hip).

249 Pointing task movements demonstrated linear displacement of the ankle dependent of the 250 adopted posture. Figure 4 illustrates the forward/backward trajectory this joint related to the 251 upward finger displacement. We observed that while seated without feet support, subjects 252 adopted a synchronous flexion of the knee (i.e. backward displacement of the ankle) with the 253 finger displacement. Indeed, a backward displacement of the ankle began $30 \mathrm{~ms} \pm 1.0$ before the 254 finger upward displacement. This pattern did not happen in the other two postures and suggests a 255 synergic pattern of lower-upper limbs, accompanying arm movement.

\section{Insert Figure 4}

Figure 4: Kinematic synergy a typical subject recorded during one single trial. Plotted signals were normalized with respect to their total movement duration.Up: Upright posture; SitSup: Sit posture with contact feet support; SitUnsup: Sit unsupported posture. A(Finger Velocity); B (Ankle displacement); C (Finger displacement).

\section{Discussion}

The present study aimed to verify whether and how APAs patterns and kinematic features

262 changes while the subject postural stability was manipulated. Indeed, two seated postures (with 263 and without feet support) and a standing posture were adopted while subjects performed an arm 264 pointing task. These different postures permit to modify the degrees of postural stability. 265 Accordingly, three postures were used, from high (i.e., sitting with feet support) to low stability 266 (i.e., standing), as well as an intermediate position with a larger base of support but without feet 267 contact that generated a higher instability than in sitting with support. The main results revealed 268 that kinematic parameters and APAs latencies are dependent of the postural chains/configuration 269 as well as the degree of stability that these last ones promote.

\section{Kinematic features}

271 Different kinematic patterns were expected due to the postural stability manipulation since 272 the base of support was limited to the feet in Up posture and defined by the buttocks in SitUnsup 273 or both buttocks and feet in SitSup posture. As previously predicted, challenging postural stability 274 induced different planning evidenced by an increase of the RT and MD for the seated condition 275 without support compared to seated with support or standing. This suggests that arm motor plan 
276 becomes more challenging in the SitUnsup posture as indicated by the greater RT that is 277 classically interpreted as an index of task difficulty (Cuisinier, Olivier \& Nougier, 2007). One 278 possibility would be that sitting without support does not give efficient mechanical mean (i.e. 279 buttock) to accurately control posture and movement, thus challenging the motor planning. This 280 is partially verified when considering the arm velocity profile which was significantly affected by 281 postural stability. In agreement with our initial prediction we found greater max velocity and 282 shorter deceleration duration of the arm pointing movement in the most stable position. Indeed, 283 the velocity profile (or ACC/MD ratio) is classically described as reflecting the content of motor 284 planning (Morasso 1982, Papaxanthis et al., 2003b). More specifically, it is known that 285 movements performed in the vertical plane used similar motor planning independently of the 286 effector, and are directly dependent of the mechanical effect of gravity force field that change 287 during upward and downward movement (Papaxanthis, Dubost \& Pozzo, 2003). Herein, our 288 result suggests that the equilibrium constraints rather than postural configuration were taken into 289 account to plan an upper arm movement. This confirms the greater difficulty to plan the task (i.e. 290 smaller pic of velocity) when a subtask (equilibrium) is added to the pointing one. Another point 291 is that, although the APA was similar between the two-seated posture (see APA features bellow), 292 the acceleration time and movement duration ratio (ACC/MD) was higher in the Sitsup posture. 293 If the role of APA was only to counteract the perturbation, we would expect to find a different 294 behavior of temporal activation between theses postures. Our results also extent a recent research 295 form Stamenkovic \&, Stapley (2016) which demonstrated that the muscle spatial recruitment was 296 in favour of assisting initiation of movement in reaching during stance(Stamenkovic \& Stapley, 297 2016).

298 Since subjects were free to modulate the kinematic features of the movement, one could 299 expect that the CNS would only use APA to minimize the perturbing effect of the dynamics of the 300 movement, as described in the classical paradigm. But, we did not observed differences in the 301 velocity of the movement between the positions in opposite of previous papers. This mean that 302 the perturbation was equal, although APA magnitude, as it will be described in the next topic, 303 were different in the upright position, compared to the other two. Previously, it was described that 304 APA magnitude is scaled with movement velocity (Bertucco \& Cesari, 2010), which was not the 305 case in our paper. This strongly suggests that APA has an additional contribution than the 306 classical paradigm. 

observed the presence of APAs independently of the posture, confirming our prediction. Stable postures may not require APAs stabilizer, but the presence of APAs regardless the stability suggests an additional role of APA beyond the feedforward control of the other body parts (i.e as

312 accelerators, or to facilitate the pointing movement). Indeed, for all postures, the activity of these

313 muscles anticipated the arm pointing movement replicating the classical deactivation of anterior 314 muscles (RF and TA) and activation of posterior muscles (SOL and ST) (Teyssedre et al., 2000;

315 Chiovetto et al., 2010). By showing the presence of APA whatever the stability condition, our 316 results demonstrated that adults performing arm movements while seated also present direction317 specific activity preceding the focal muscle. This supports the idea that APAs may be used either 318 to facilitate the task and/or to control the postural instability by activation of the same trunk 319 muscles in different feedforward patterns (Aruin \& Shiratori, 2003a) according to the postural 320 configuration (i.e. sitting vs. standing posture).

Another point is that, although the APA was similar between the two seated posture, the acceleration time and movement duration ratio (ACC/MD) was higher in the Sitsup posture, which indicates a greater facility to plan the movement in this situation. If the role of APA was only counteract the perturbation, we would expect to find a different behavior of temporal activation between theses postures.

However, even if we observed APAs for all postures, the temporal pattern of these APAs differed and was dependent of the postural chains/configuration. While the thigh muscles (ST and RF) were activated earlier than the lower leg muscles (SOL and TA) in Up posture, the order was inverted when seated (both, with or without support). Therefore, our results demonstrated that sitting posture, regardless the stability, was associated with APAs in a different pattern compared to standing.

In standing posture the earlier events are located on the proximal muscle RF and ST while in sitting this is the distal one (TA). Anticipation of ST in the Up posture, appears to be related to its role in the hip extension in order to counteract the forces on the pelvis produced by the reaching task and producing a trunk forward tilting movement (Hodges, Cresswell \& 336 Thorstensson, 1999; Pozzo, Ouamer \& Gentil, 2001). Indeed, the seated subjects had the pelvis 337 stabilized by the natural postural chain (Forssberg \& Hirschfeld, 1994; Van Der Fits et al., 1998; 338 Le Bozec \& Bouisset, 2004; Cuisinier, Olivier \& Nougier, 2007; Vette et al., 2010) and ST acted 339 as knee flexor instead of hip flexor and also activated after the lower leg muscles. This is 
340 supported by our kinematic data about the SitUnsup posture that highlighted a simultaneous 341 upward finger (arm) and backward ankle displacement. We propose that this kinematic profile 342 reflects a strategy dedicated to facilitating the upper-limb movement in this posture. Two 343 assumptions may be related to that. First, the initial muscle tone is different from standing to 344 sitting postures (i.e. different joint positions, muscle length) and thus the following activity of 345 these muscles change with respect to the resting state of the muscles. Second, the upward 346 acceleration of the arm produces a forward trunk tilting that can be counteracted when sitting 347 with support but is more difficult when no support is added on the feet. So, APAs not only serves 348 the whole-body equilibrium to the movement initiation, but are also concerned to provide the correct postural set to obtain the correct movement(Cavallari et al., 2016).

The APA magnitude of SOL and ST are in line with the literature that states that more APA is necessary to counterbalance the disturbances under more challenge stability situations.

352 For RF muscle, Sit Unsup posture showed even lower magnitude compared to the other postures, 353 and this may be related to the ankle displacement observed in this position (i.e.activation of the antagonist, ST). Surprisingly, TA did not show this behavior, and had higher magnitude in SitSup position. This refute the reasonable that APA magnitude is strictly related to the mechanical perturbation. Although we did not record the center of pressure (COP) data, maybe this muscle had some participation in COP displacement, accompanying the movement, in forward direction. This may not have happened in Sit Unsup due to kinematic pattern imposed by lack of feet contact and the ankle backward displacement.

Contrarily to SitUnsup posture, the SitSup did not present knee displacement due to the constraint of feet contact with the floor. However, the EMG pattern between both sitting posture was the same, since the postural chain was not modified. We also found that the TA activity appeared earlier for the seated postures compared to standing posture, acting as an ankle dorsiflexor(Erim et al., 1996; Aruin \& Shiratori, 2003a; van der Heide et al., 2003). This early activity of TA in standing posture did not happen since the mechanical load in this joint is totally 366 different and the body weight is completely loaded over it, delaying its participation. The fact that we observed different APA patterns between the sitting postures and the standing condition and not between the stability levels confirm our predictions (see supplementary table). Thus, our results strongly suggest that APA acts to facilitate the limb movement and to counteract perturbation forces. Kinematics features, on the other hand, seem to reflect less challenging task

371 and simple motor plan when the body is stabilized. 


\section{Conclusion}

The present study showed that the APAs seem to be related to the stability conditions

since we observed differences of EMG features for the two seated postures whereas the

the motor planning of the upper-limb during a pointing task whereas the muscle postural chain

(sitting vs. standing) was also determinant for APAs.

\section{References}

381

382

383

384

385

386

387

388

389

390

391

392

393

394

395

396

397

398

399

400

401

402

403

404

405

406

407

408

Aruin A., Shiratori T. 2003a. Anticipatory postural adjustments while sitting: The effects of different leg supports. Experimental Brain Research 151:46-53. DOI: 10.1007/s00221-0031456-y.

Aruin A., Shiratori T. 2003b. Anticipatory postural adjustments while sitting: The effects of different leg supports. Experimental Brain Research 151:46-53. DOI: 10.1007/s00221-0031456-y.

Berret B., Bonnetblanc F., Papaxanthis C., Pozzo T. 2009. Modular control of pointing beyond arm's length. The Journal of neuroscience : the official journal of the Society for Neuroscience 29:191-205. DOI: 10.1523/JNEUROSCI.3426-08.2009.

Bertucco M., Cesari P. 2010. Does movement planning follow Fitts' law? Scaling anticipatory postural adjustments with movement speed and accuracy. Neuroscience 171:205-213. DOI: 10.1016/j.neuroscience.2010.08.023.

Bonnetblanc F., Martin O., Teasdale N. 2004. Pointing to a target from an upright standing position: Anticipatory postural adjustments are modulated by the size of the target in humans. Neuroscience Letters 358:181-184. DOI: 10.1016/j.neulet.2004.01.020.

Le Bozec S., Bouisset S. 2004. Does postural chain mobility influence muscular control in sitting ramp pushes? Experimental Brain Research 158:427-437. DOI: 10.1007/s00221-004-1918$\mathrm{x}$.

Cavallari P., Bolzoni F., Bruttini C., Esposti R. 2016. The Organization and Control of Intra-Limb Anticipatory Postural Adjustments and Their Role in Movement Performance. Frontiers in Human Neuroscience 10. DOI: 10.3389/fnhum.2016.00525.

Cecchi M., Došlă Z., Marini M. 2001. Anticipatory postural adjustments during load catching by standing subjects. Clinical Neurophysiology 112:1250-1265. DOI: 10.1016/S13882457(01)00553-3.

Chikh S., Watelain E., Faupin A., Pinti A., Jarraya M., Garnier C. 2016. Adaptability and Prediction of Anticipatory Muscular Activity Parameters to Different Movements in the Sitting Position. Perceptual and Motor Skills 123:190-231. DOI: 
409

410

411

412

413

414

415

416

417

418

419

420

421

422

423

424

425

426

427

428

429

430

431

432

433

434

435

436

437

438

439

440

441

442

443

444

445

446

447

448

449

450

451

452

453

454

455

456

457

Chiovetto E., Berret B., Pozzo T. 2010. Tri-dimensional and triphasic muscle organization of whole-body pointing movements. Neuroscience 170:1223-1238. DOI: 10.1016/j.neuroscience.2010.07.006.

Cholewicki J., Polzhofer GK., Radebold A. 2000. Postural control of trunk during unstable sitting. Journal of Biomechanics 33:1733-1737. DOI: 10.1016/S0021-9290(00)00126-3.

Cuisinier R., Olivier I., Nougier V. 2007. The increased foreperiod duration to attain the neutral optimal preparation from sitting to standing. Experimental Brain Research 180:321-331. DOI: 10.1007/s00221-007-0862-y.

Erim Z., De Luca CJ., Mineo K., Aoki T. 1996. Rank-ordered regulation of motor units. Muscle Nerve 19:563-573.

Van Der Fits IBM., Klip AWJ., Van Eykern LA., Hadders-Algra M. 1998. Postural adjustments accompanying fast pointing movements in standing, sitting and lying adults. Experimental Brain Research 120:202-216. DOI: 10.1007/s002210050394.

Forssberg H., Hirschfeld H. 1994. Postural adjustments in sitting humans following external perturbations: muscle activity and kinematics. Experimental brain research. Experimentelle Hirnforschung. Experimentation cerebrale 97:515-527. DOI: 10.1007/BF00241545.

van der Heide JC., Otten B., van Eykern L a., Hadders-Algra M. 2003. Development of postural adjustments during reaching in sitting children. Experimental brain research 151:32-45. DOI: $10.1007 / \mathrm{s} 00221-003-1451-3$.

Hermens HJ., Freriks B., Disselhorst-Klug C., Rau G. 2000. Development of recommendations for SEMG sensors and sensor placement procedures. Journal of Electromyography and Kinesiology 10:361-374. DOI: 10.1016/S1050-6411(00)00027-4.

Hodges P., Cresswell A., Thorstensson A. 1999. Preparatory trunk motion accompanies rapid upper limb movement. Experimental Brain Research 124:69-79. DOI: $10.1007 / \mathrm{s} 002210050601$.

Juras G., Słomka K. 2013. Anticipatory postural adjustments in dart throwing. Journal of human kinetics 37:39-45. DOI: 10.2478/hukin-2013-0023.

Moore S., Brunt D. 1991. Effects of trunk support and target distance on postural adjustments prior to a rapid reaching task by seated subjects. Arch Phys Med Rehabil 72:638-641. DOI: 0003-9993(91)90204-V [pii].

Moore S., Brunt D., Nesbitt ML., Juarez T. 1992. Investigation of evidence for anticipatory postural adjustments in seated subjects who performed a reaching task. Physical therapy 72:335-343.

Papaxanthis C., Dubost V., Pozzo T. 2003. Similar planning strategies for whole-body and arm movements performed in the sagittal plane. Neuroscience 117:779-783.

Papaxanthis C., Pozzo T., Schieppati M. 2003. Trajectories of arm pointing movements on the sagittal plane vary with both direction and speed. Experimental brain research. Experimentelle Hirnforschung. Experimentation cerebrale 148:498-503. DOI: 10.1007/s00221-002-1327-y.

Pozzo T., Ouamer M., Gentil C. 2001. Simulating mechanical consequences of voluntary movement upon whole-body equilibrium : the arm-raising paradigm revisited. 49:39-49.

Santos MJ., Kanekar N., Aruin AS. 2010. The role of anticipatory postural adjustments in compensatory control of posture: 2. Biomechanical analysis. Journal of Electromyography and Kinesiology 20:398-405. DOI: 10.1016/j.jelekin.2010.01.002.

Sijper H., Latash M. 2000. The effects of instability and additional hand support on anticipatory postural adjustments in leg, trunk, and arm muscles during standing. Experimental Brain Research 135:81-93. DOI: 10.1007/s002210000492.

Stamenkovic A., Stapley PJ. 2016. Trunk muscles contribute as functional groups to directionality of reaching during stance. Experimental Brain Research 234:1119-1132. DOI:

Peer] reviewing PDF | (2017:09:20383:2:0:ACCEPTED 8 Jan 2018) 
458

459

460

461

462

463

464

465

466

467

468

469

470

471

472

473

474

475

476
10.1007/s00221-015-4536-X.

Stapley PJ., Pozzo T., Cheron G., Grishin A. 1999. Does the coordination between posture and movement during human whole-body reaching ensure center of mass stabilization? Experimental Brain Research 129:134-146. DOI: 10.1007/s002210050944.

Stapley P., Pozzo T., Grishin a. 1998. The role of anticipatory postural adjustments during whole body forward reaching movements. Neuroreport 9:395-401. DOI: 10.1097/00001756199802160-00007.

Teyssedre C., Lino F., Zattara M., Bouisset S. 2000. Anticipatory EMG patterns associated with preferred and non-preferred arm pointing movements. Experimental Brain Research 134:435-440. DOI: 10.1007/s002210000490.

Tijtgat P., Vanrenterghem J., Bennett SJ., De Clercq D., Savelsbergh GJP., Lenoir M. 2013. Postural adjustments in catching: on the interplay between segment stabilization and equilibrium control. Motor control 17:48-61.

Vette AH., Masani K., Sin V., Popovic MR. 2010. Posturographic measures in healthy young adults during quiet sitting in comparison with quiet standing. Medical Engineering and Physics 32:32-38. DOI: 10.1016/j.medengphy.2009.10.005.

Yoshida S., Nakazawa K., Shimizu E., Shimoyama I. 2008. Anticipatory postural adjustments modify the movement-related potentials of upper extremity voluntary movement. Gait and Posture 27:97-102. DOI: 10.1016/j.gaitpost.2007.02.006. 
Figure 1

Experimental pointing task.

View of the experimental set-up for the task showing a participant in final posture and the three possible targets. The central diode of the bar between the two laterals was situated exactly in front of the participants' right shoulder. Participants were asked to point their index finger at the central diode which suddenly turned on. Postures: A(Up); B (SiUnsup); C (SitSup).

A

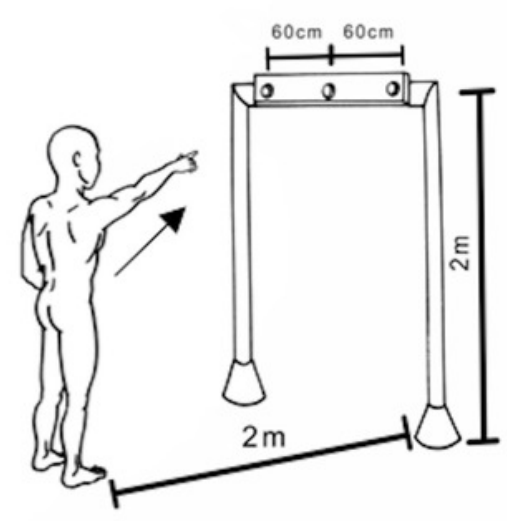

B

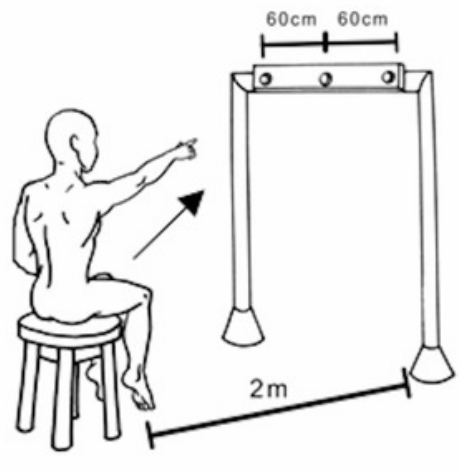

$\mathrm{C}$

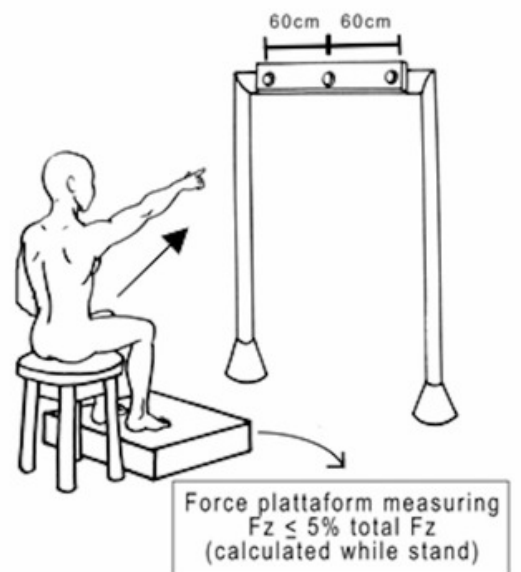




\section{Figure 2}

Raw muscle activity of a typical subject recorded during one single trial.

Plotted signals were just rectified and normalized with respect to their maximum values recorded over all trials. The vertical dashed line mark finger movement onset (tzero). The time interval between 300 ms before movement onset was considered.Up: Upright posture; SitSup: Sit posture with contact feet support; SitUnsup: Sit unsupported posture; Muscles: DEL: Deltoideous; ST: Semitendinosus; RF: Rectus Femoris; SOLSoleous; TA: Tibialis Anterior.
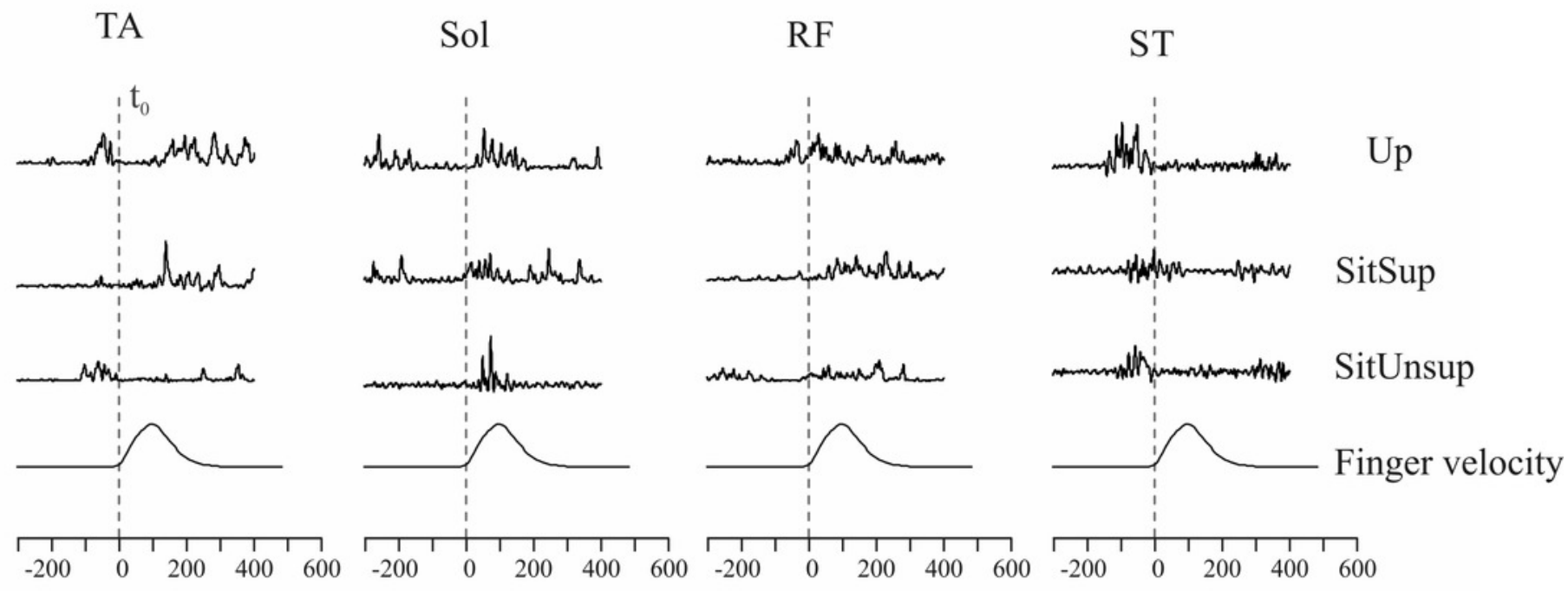

Time (ms) 


\section{Figure 3}

Latency (activation/deactivation before tzero) for lower limb muscles.

Up: Upright posture; SitSup: Sit posture with contact feet support; SitUnsup: Sit unsupported posture; Muscles: DEL: Deltoideous; ST: Semitendinosus; RF: Rectus Femoris; SOLSoleous; TA: Tibialis Anterior. ${ }^{*} p<0,05$ two way anova and post hoc test (differences between SIT SUP $x$ UP and SIT UNSUP $x$ UP). 


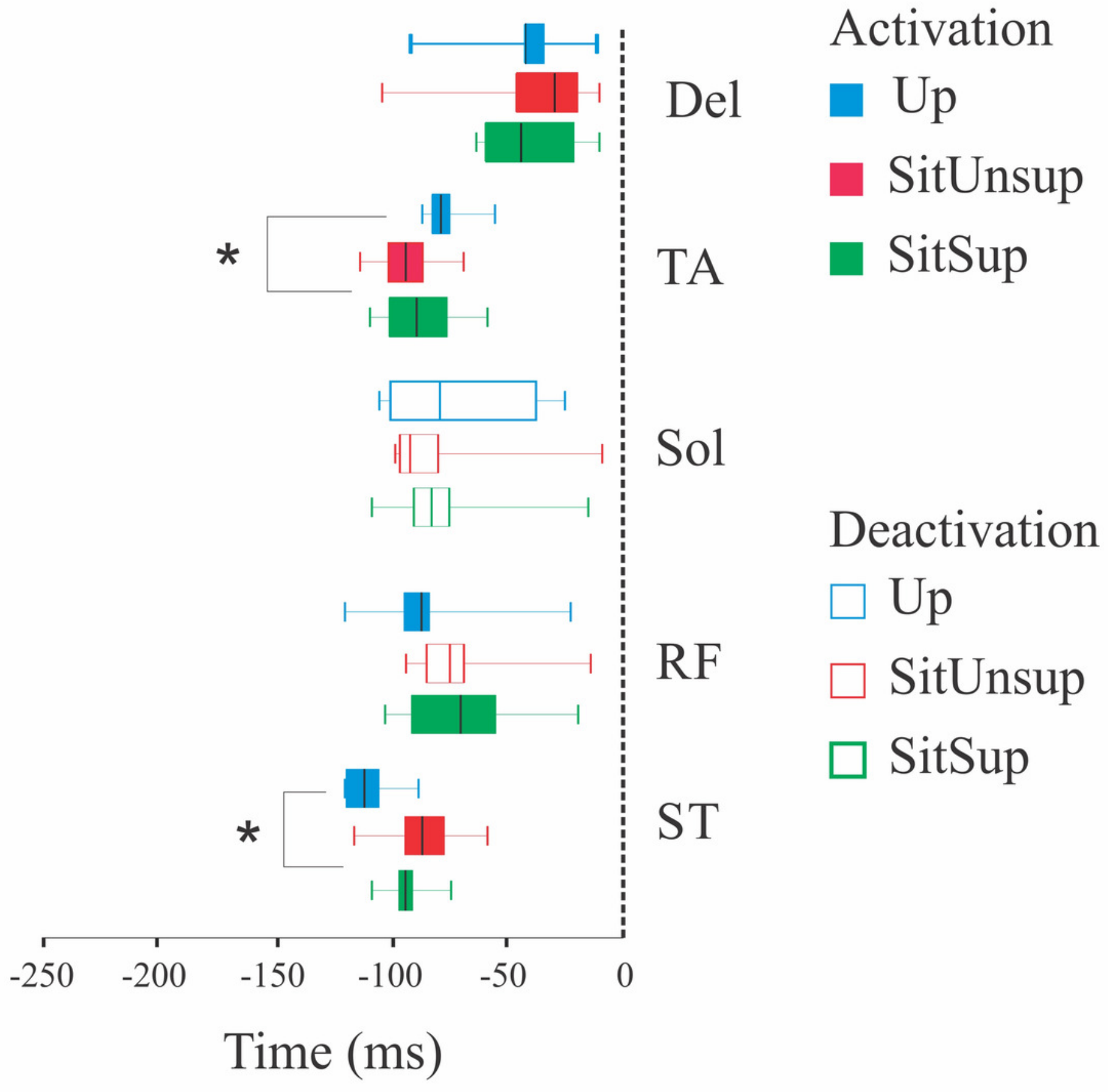




\section{Figure 4}

Kinematic synergy a typical subject recorded during one single trial.

Plotted signals were normalized with respect to their total movement duration. Up: Upright posture; SitSup: Sit posture with contact feet support; SitUnsup: Sit unsupported posture. A(Finger Velocity); B (Ankle displacement); C (Finger displacement). 

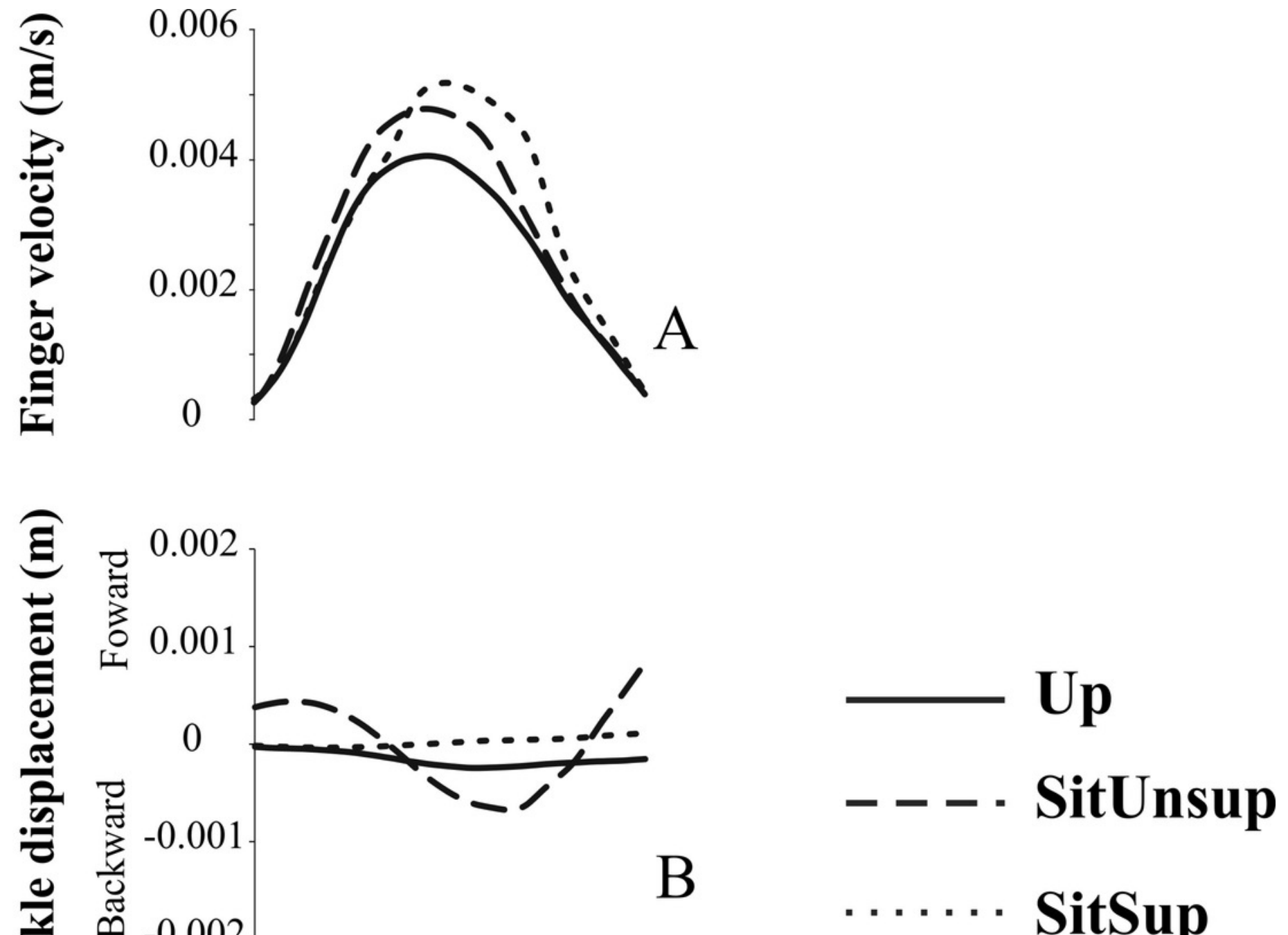

B
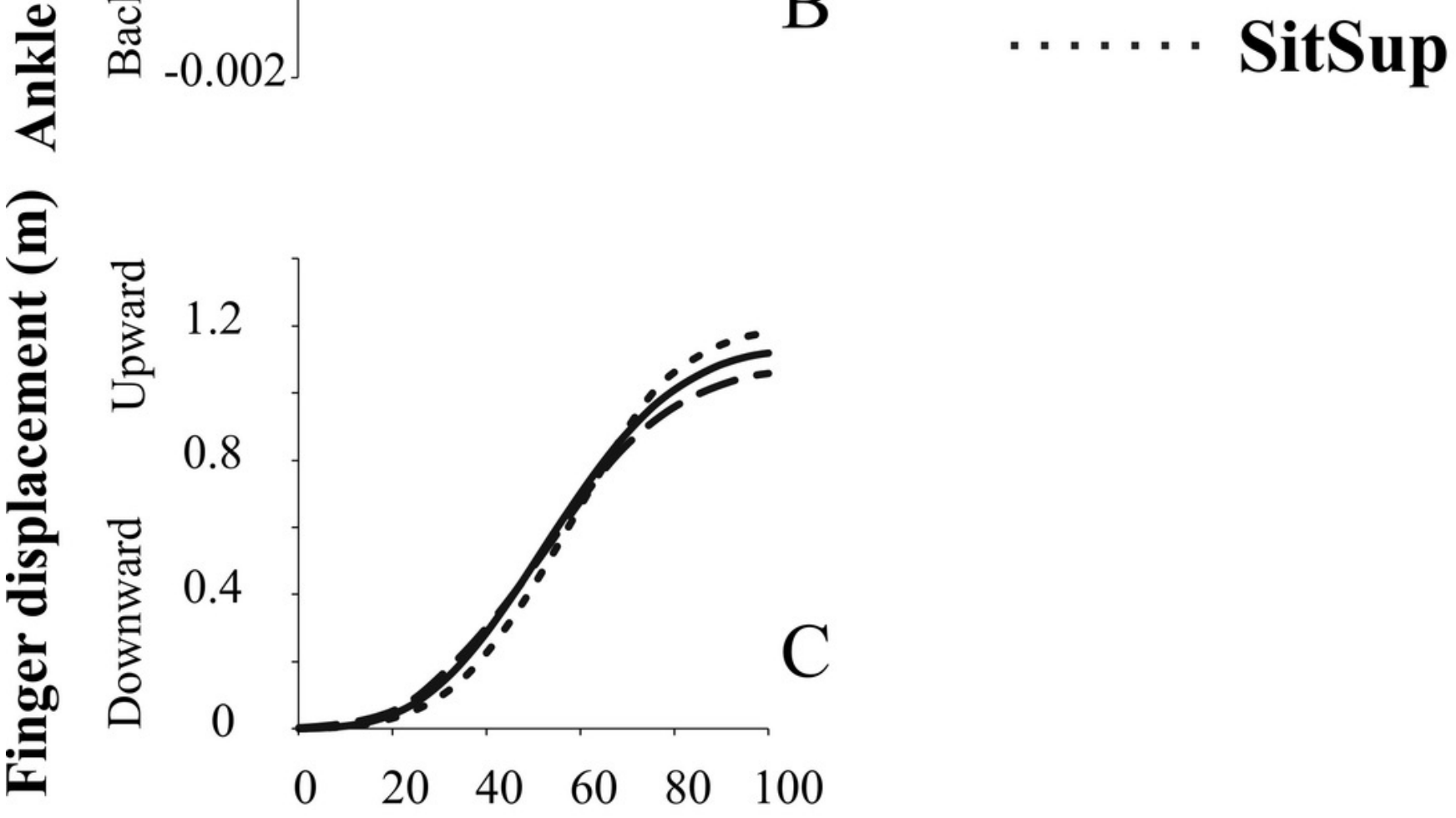

Movement duration (\%) 


\section{Table $\mathbf{1}$ (on next page)}

Comparison between kinematic parameters.

Mean values kinematic parameters. Values are given as mean (SD). * $p<0.005$ difference between Sit Unsup and the two other postures. ${ }^{* *} p<0.005$ difference between Sit Sup and the two other postures. 
Table 1. Comparison between Kinematic parameters.

\begin{tabular}{|c|c|c|c|c|c|}
\hline & $\begin{array}{c}\text { Finger } \\
\text { displacement } \\
(\mathrm{cm})\end{array}$ & $\begin{array}{l}\text { Reaction Time } \\
\text { (ms) }\end{array}$ & $\begin{array}{l}\text { Movement } \\
\text { duration } \\
(\mathrm{ms})\end{array}$ & $\begin{array}{l}\text { Velocity } \\
(\mathrm{m} / \mathrm{s})\end{array}$ & $\begin{array}{c}\text { Acceleration } \\
\text { time/ } \\
\text { Movement } \\
\text { duration }\end{array}$ \\
\hline $\begin{array}{l}\text { Sit } \\
\text { Sup }\end{array}$ & $118.74(6.13)$ & $407.31(21.03)$ & $408.12(68.72)$ & $\begin{array}{c}0.48 \\
(0.03)\end{array}$ & $0.46(0.06) * *$ \\
\hline $\begin{array}{l}\text { Sit } \\
\text { Unsun }\end{array}$ & $116.90(8.22)$ & $433.71(13.07)^{*}$ & $529.06(127.71)^{*}$ & 0.46 & $0.38(0.04)$ \\
\hline $\begin{array}{l}\text { Unsup } \\
\text { Up }\end{array}$ & $116.48(7.83)$ & $411.01(18.50)$ & $444.56(106.64)$ & $\begin{array}{c}(0.03) \\
0.45 \\
(0.02)\end{array}$ & $0.37(0.03)$ \\
\hline
\end{tabular}




\section{Table 2 (on next page)}

Comparison between normalized integrated electromyographic activity (EMGi).

Normalized integrated electromyographic activity (EMGi) of muscles Mean values integral parameters (\%). Values are given as mean (SD). $* p<0.005$ difference between Up and the two other postures; ${ }^{* *} p<0.005$ difference between Sit Sup and the two other postures; ${ }^{p} p$ $<0.005$ difference between Sit Unsup and the two other postures. 
Table 2. Comparison between normalized integrated electromyographic activity (EMGi).

\begin{tabular}{lccc|c}
\hline & $\mathrm{TA}(\%)$ & $\mathrm{SOL}(\%)$ & $\mathrm{RF}(\%)$ & $\mathrm{ST}(\%)$ \\
Sit Sup & $67.39(27.30)$ & $52.11(28.12)$ & $45.07(20.85)$ & $40.08(28.21)$ \\
& $* *$ & & & \\
Sit Unsup & $45.37(35.02)$ & $50.87(30.63)$ & $24.40(20.20)^{\#}$ & $40.43(28.63)$ \\
Up & $49.52(32.04)$ & $75.59(15.26) *$ & $57.27(27.73)$ & $55.84(23.92) *$ \\
\hline
\end{tabular}

Normalized integrated electromyographic activity (EMGi) of muscles Mean values integral parameters (\%). Values are given as mean (SD). ${ }^{*} \mathrm{p}<0.005$ difference between Up and the two other postures; ${ }^{* *} \mathrm{p}<0.005$ difference between Sit Sup and the two other postures; ${ }^{\#} \mathrm{p}<0.005$ difference between Sit Unsup and the two other postures. 